\title{
Sinus surgery improves lower respiratory tract reactivity during aspirin desensitization for AERD
}

\author{
George X. Huang, MD ${ }^{\mathrm{a}}$, Marina L. Palumbo, BA ${ }^{\mathrm{b}}$, Joseph I. Singer, BA ${ }^{\mathrm{b}}$, Katherine N. Cahill, \\ $M^{c}$, Tanya M. Laidlaw, MD $^{b}$ \\ aDepartment of Medicine, Brigham and Women's Hospital, Boston, Mass \\ bDivision of Rheumatology, Immunology and Allergy, Brigham and Women's Hospital, Boston, \\ Mass \\ 'Division of Allergy, Pulmonary and Critical Care Medicine, Vanderbilt University Medical Center, \\ Nashville, Tenn
}

\section{TO THE EDITOR:}

Aspirin desensitization followed by maintenance therapy with daily high-dose aspirin has been used clinically to control symptoms in patients with aspirin-exacerbated respiratory disease (AERD) since the early 1980s. ${ }^{1}$ Prospective studies have demonstrated that treatment with high-dose aspirin leads to long-term improvements in upper and lower respiratory tract symptoms as well as reduction in corticosteroid requirement in patients with AERD. ${ }^{2,3}$ Although generally safe, aspirin challenges and desensitizations do carry a risk of precipitating severe asthma attacks and occasionally systemic reactions. Although no deaths due to physician-observed aspirin desensitization have ever been recorded, ${ }^{4}$ it is recommended that emergency medical resources be readily available should intensive care be needed.

Few studies have examined factors that influence the safety of aspirin challenges and desensitizations for AERD. In the literature, leukotriene receptor antagonists such as montelukast are reported to reduce the severity of lower respiratory tract reactions and have mixed efficacy in reducing aspirin-induced upper airway reactions during aspirin desensitization. ${ }^{5,6}$ Therefore, it is common practice for patients to be premedicated with montelukast before aspirin desensitization. Investigation of other factors that influence the severity of aspirin-induced reactions during desensitization, such as timing of functional endoscopic sinus surgery (FESS) or nasal polyp burden, could ultimately allow for improved patient safety. Aspirin desensitization is often performed after FESS. The rationale for this is 2-fold: (1) high-dose aspirin maintenance after FESS can delay or obviate the need for revision sinus surgery and prolong the benefits of surgery for these patients and (2) aspirin use is contraindicated during FESS and desensitization would need to be repeated following surgical intervention. ${ }^{7,8}$ Recently, Jerschow et $\mathrm{al}^{9}$ reported that, in a predominantly African-

Corresponding author: Tanya M. Laidlaw, MD, Brigham and Women's Hospital, 60 Fenwood Rd, Building of Transformative Medicine, Rm 5002M, Boston, MA 02115. tlaidlaw@bwh.harvard.edu.

Conflicts of interest: The authors declare that they have no relevant conflicts of interest. 
American and Hispanic/Latino population, aspirin-induced reactions were less severe during desensitizations that occurred shortly after FESS. In this study, we applied similar methodology to a more generalized population. We hypothesized that the inflammatory milieu within the nasal polyp tissue may contribute to the severity of aspirin-induced reactions, and therefore suspected that there would be less severe aspirin-induced lower respiratory tract reactions when a recent FESS precedes aspirin desensitization.

In this retrospective case series, adult subjects with AERD were identified who had undergone 2 oral aspirin challenges or desensitizations at Brigham and Women's Hospital, 1 within 60 days after FESS and 1 in the absence of recent FESS. Aspirin desensitizations were conducted using a single-day oral challenge protocol. ${ }^{10}$ Reasons for more than 1 aspirin desensitization included an intervening pregnancy or surgery that required stoppage of aspirin. Subjects with incomplete spirometry data were excluded. Aspirin-induced changes in $\mathrm{FEV}_{1}$ were compared for the aspirin challenges following FESS or without recent FESS using the paired student $t$ test. Available baseline asthma control test (ACT) data were compared using the paired student $t$ test. Additional end points included physician-recorded reports of aspirin-induced upper airway, gastrointestinal, and skin symptoms, which were compared using chi-square analysis. Provocative aspirin dose during aspirin desensitization presurgery compared to postsurgery was also examined. Odds ratios were calculated for developing a clinically significant lower airway reaction (defined as $>15 \%$ decrease in $\mathrm{FEV}_{1}$ from baseline) and for developing any clinically apparent reaction (defined as aspirin-induced lower airway and/or upper airway symptoms) during desensitization. All research was approved by the Institutional Review Board of Partners Healthcare.

Sixteen subjects met inclusion criteria (Table 1). The frequency of grossly visible nasal polyps, as assessed by the allergist on intranasal otoscopic examination, was lower postsurgery (53\% vs $0 \% ; P=.002$ ). Baseline $\mathrm{FEV}_{1}$ was similar presurgery versus postsurgery (91.6\% vs $88.5 \%$ predicted; $P=.30 ; \mathrm{n}=16)$. Baseline ACT score was slightly higher postsurgery ( 21 vs $23 ; P=.02 ; \mathrm{n}=9$ ). In the 3 patients for whom they were recorded, there was a trend toward lower baseline Sinonasal Outcome Test-22 score postsurgery ( 7 vs $38 ; P=.06 ; \mathrm{n}=3)$. In terms of lower airway symptoms, FESS within 60 days was associated with less decline in $\mathrm{FEV}_{1}$ during aspirin desensitization/challenge (15.2\% vs $8.2 \%$ decrease; $P=.030 ; \mathrm{n}=16$; Figure 1). When subjects on oral corticosteroids and/or montelukast postsurgery but not presurgery were excluded, the difference in decline in $\mathrm{FEV}_{1}$ postsurgery (14.6\% decrease vs $4.2 \%$ decrease; $P=.027 ; \mathrm{n}=7$ ) remained. Furthermore, 6 of 16 subjects (37.5\%) had abrogation of significant lower airway reactivity during postsurgery desensitization. The odds ratio of having a significant lower respiratory tract reaction presurgery compared with postsurgery was $7(P=.022 ; \mathrm{n}=16)$. In terms of other symptoms, subjects who were postsurgery had decreased rates of aspirin-induced upper airway symptoms compared with presurgery subjects ( $94 \%$ vs $63 \% ; P=.033 ; \mathrm{n}=16)$, but there was no difference in the rate of aspirin-induced gastrointestinal symptoms (13\% vs $19 \% ; P=.63$; $\mathrm{n}=16)$ or aspirin-induced skin symptoms ( $13 \%$ vs $25 \% ; P=.37 ; \mathrm{n}=16)$. Altogether, 6 of 16 subjects (38\%) showed no reaction at all and therefore had a clinically silent desensitization postsurgery. The odds ratio of having a clinically apparent aspirin-induced reaction presurgery compared with postsurgery was $9(P=.033 ; \mathrm{n}=16$; Table 1$)$. For 
patients who developed reactions during both their presurgery and postsurgery desensitizations, there was no difference in the provocative dose of aspirin.

A limitation of this study is that not all patients remained on high-dose aspirin following their challenge/desensitization, so the therapeutic efficacy of high-dose aspirin could not be evaluated. Another limitation of this study is the relatively small sample size. Baseline Sinonasal Outcome Test-22 score postsurgery was not significantly decreased compared with presurgery in this study population, in contrast to previous reports, ${ }^{3}$ likely due to the small data set available. Although there was some increase in ACT score postsurgery, for the safety of our patients, their asthma was well controlled before the scheduled aspirin desensitization and a difference of this magnitude may not be clinically significant. ${ }^{11}$ In this study, we observed that FESS before aspirin desensitization is associated with diminished aspirin-induced lower airway reactions during aspirin challenge/desensitization in patients with AERD. This finding is similar to that in the recent work by Jerschow et al. ${ }^{9}$ From a physiologic perspective, this suggests that nasal polyp tissue contributes to the severity of aspirin-induced reactions. In practice, this finding has relevance for patient safety and selecting the optimal timing of aspirin desensitization for AERD. Furthermore, we report that nasal sinus surgery can also lead to clinically silent desensitization, characterized by complete lack of upper airway, lower airway, gastrointestinal, and skin reactions. This finding also has clinical implications in that it suggests that aspirin challenges for diagnosis of AERD may be falsely negative if they are only performed immediately following nasal sinus surgery. Further work elucidating how nasal polyp tissue communicates with the lower respiratory system is warranted. Previous studies suggest that this may be due to alterations in serum prostaglandin D2 and lipoxin A4 caused by FESS, ${ }^{9}$ but further studies are warranted. On a clinical practice level, future analysis could seek to identify whether there is an optimally low level of polyp burden that predicts uneventful aspirin desensitization, thereby identifying patients with AERD who should undergo nasal sinus surgery before aspirin desensitization.

\section{Acknowledgments}

This work was supported by the National Institutes of Health (grant nos. R01HL128241, K23AI118804, and U19AI070535) and by generous contributions from the Vinik and Kaye Families.

\section{REFERENCES}

1. White AA, Stevenson DD. Aspirin-exacerbated respiratory disease. N Engl J Med 2018;379:106070. [PubMed: 30207919]

2. Berges-Gimeno MP, Simon RA, Stevenson DD. Long-term treatment with aspirin desensitization in asthmatic patients with aspirin-exacerbated respiratory disease. J Allergy Clin Immunol 2003;111:180-6. [PubMed: 12532116]

3. Adappa ND, Ranasinghe VJ, Trope M, Brooks SG, Glicksman JT, Parasher AK, et al. Outcomes after complete endoscopic sinus surgery and aspirin desensitization in aspirin-exacerbated respiratory disease. Int Forum Allergy Rhinol 2018;8:49-53. [PubMed: 29105347]

4. Stevenson DD. Aspirin desensitization in patients with AERD. Clin Rev Allergy Immunol 2003;24:159-68. [PubMed: 12668896]

5. Stevenson DD, Simon RA, Mathison DA, Christiansen SC. Montelukast is only partially effective in inhibiting aspirin responses in aspirin-sensitive asthmatics. Ann Allergy Asthma Immunol 2000;85:477-82. [PubMed: 11152169] 
6. Lee DKC, Haggart K, Robb FM, Lipworth BJ. Montelukast protects against nasal lysine-aspirin challenge in patients with aspirin-induced asthma. Eur Respir J 2004;24:226-30. [PubMed: 15332389]

7. Rozsasi A, Polzehl D, Deutschle T, Smith E, Wiesmiller K, Riechelmann H, et al. Long-term treatment with aspirin desensitization: a prospective clinical trial comparing 100 and $300 \mathrm{mg}$ aspirin daily. Allergy 2008;63:1228-34. [PubMed: 18699939]

8. McMains KC, Kountakis SE. Medical and surgical considerations in patients with Samter's triad. Am J Rhinol 2006;20:573-6. [PubMed: 17181095]

9. Jerschow E, Edin ML, Chi Y, Hurst B, Abuzeid WM, Akbar NA, et al. Sinus surgery is associated with a decrease in aspirin-induced reaction severity in AERD patients. J Allergy Clin Immunol Pract 2019;7:1580-8. [PubMed: 30580047]

10. DeGregorio GA, Singer J, Cahill KN, Laidlaw T. A 1-day, 90-minute aspirin challenge and desensitization protocol in aspirin-exacerbated respiratory disease. J Allergy Clin Immunol Pract 2019;7:1174-80. [PubMed: 30391549]

11. Schatz M, Kosinski M, Yarlas AS, Hanlon J, Watson ME, Jhingran P. The minimally important difference of the Asthma Control Test. J Allergy Clin Immunol 2009;124:719-723.e1. [PubMed: 19767070] 


\section{Clinical Implications}

- In patients with aspirin-exacerbated respiratory disease, when a recent functional endoscopic sinus surgery precedes aspirin desensitization, less aspirin-induced lower airway reactivity and improved safety are observed. 


\section{$P=0.03$}

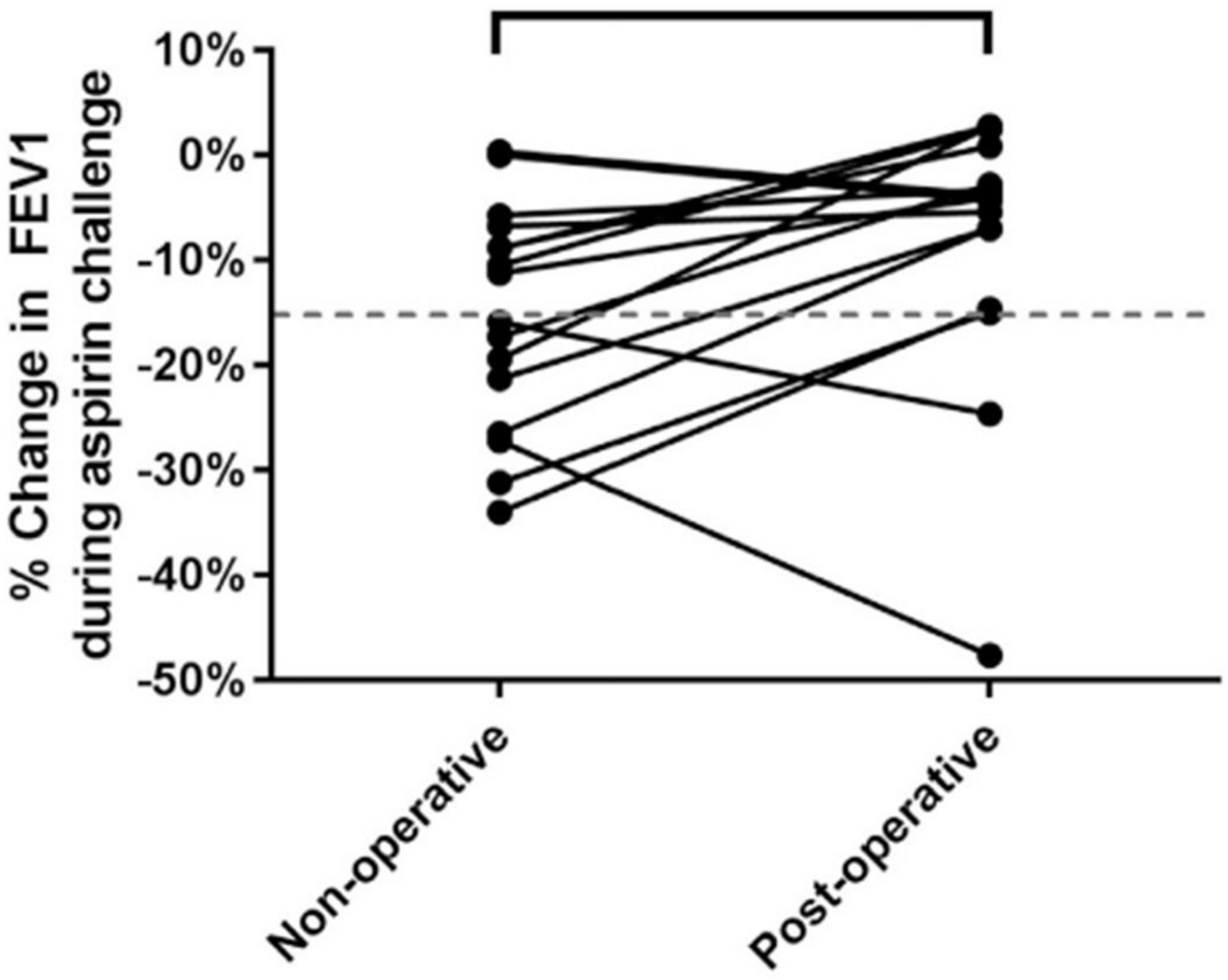

FIGURE 1.

Maximum percent change in $\mathrm{FEV}_{1}$ during aspirin challenge/desensitizations that occurred in the absence of recent sinus surgery (nonoperative), or within 60 days following sinus surgery (postoperative) $(\mathrm{N}=16)$. 


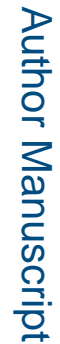

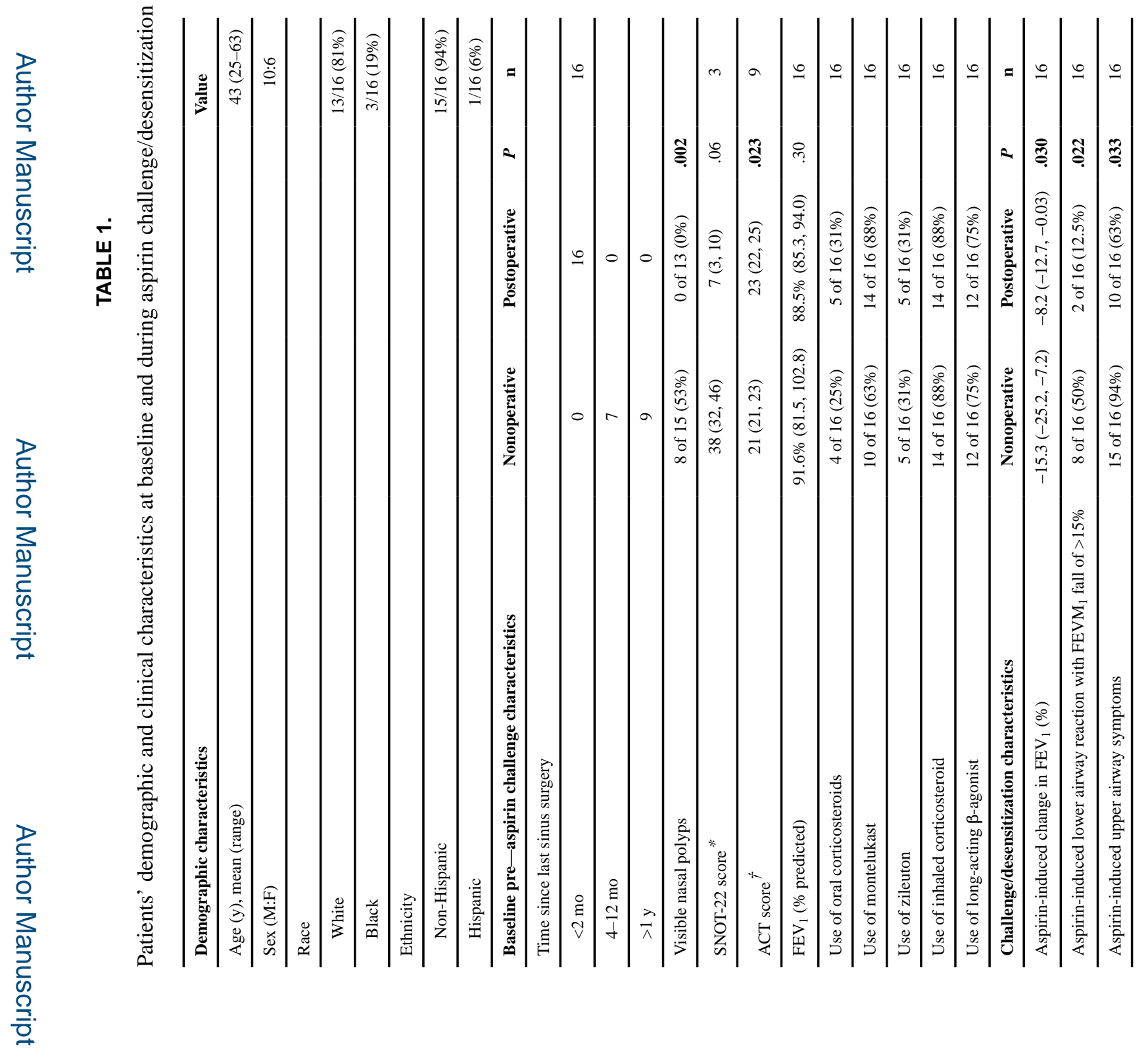


Huang et al.

Page 8
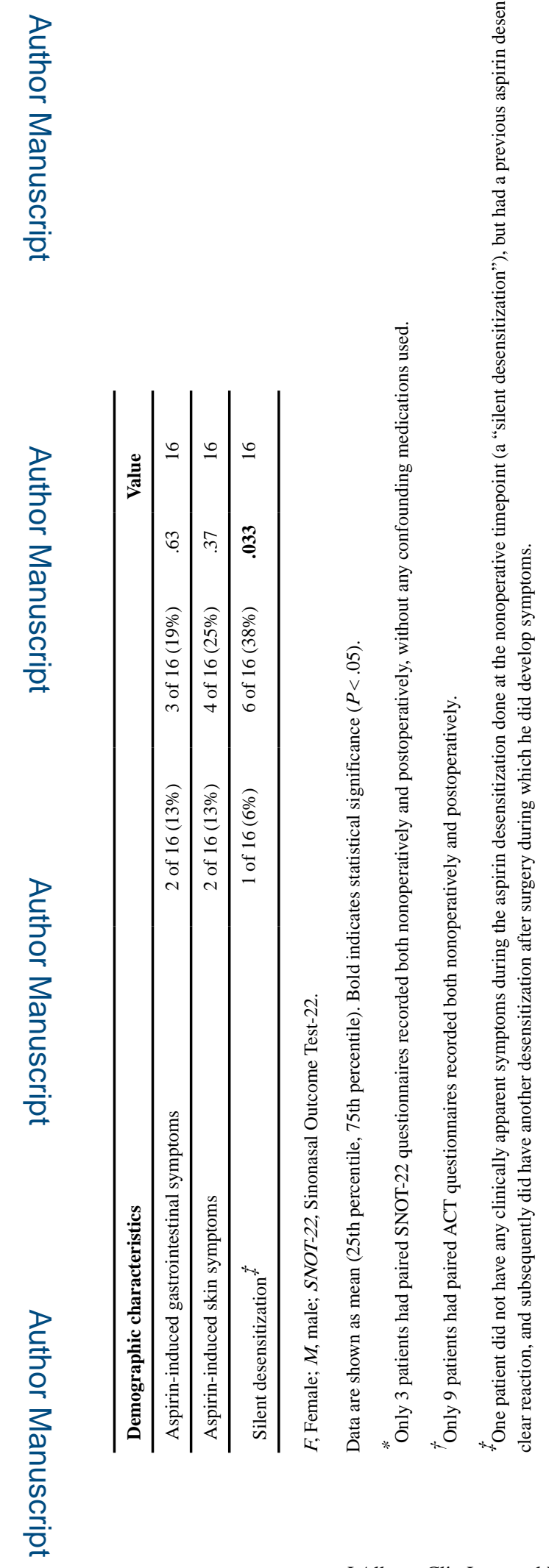

J Allergy Clin Immunol Pract. Author manuscript; available in PMC 2020 April 01. 